\title{
Breast cancer patients with low estrogen receptor expression gain no significant survival benefit from endocrine therapy: a real-world study from China
}

\author{
Shuqian Wang ${ }^{1}$, Jianbin $\mathrm{Li}^{2}$, Zefei Jiang ${ }^{2}$, Yongmei Yin ${ }^{3}$, Yunjiang Liu ${ }^{4}$, Haibo Wang ${ }^{5}$, Yue-e Teng ${ }^{6}$, Li Ma ${ }^{4}$, \\ Yuhua Song ${ }^{5}$, Peifen Fu ${ }^{1}$ \\ ${ }^{1}$ Department of Breast Cancer, The First Affiliated Hospital, Zhejiang University, School of Medicine, Hangzhou, China; ${ }^{2}$ Department of Breast \\ Cancer, The Fifth Medical Center of Chinese PLA General Hospital, Beijing, China; ${ }^{3}$ Department of Breast Cancer, Jiangsu Province Hospital, \\ Nanjing, China; ${ }^{4}$ Department of Breast Cancer, Hebei Medical University Fourth Affiliated Hospital, Shijiazhuang, China; ${ }^{5}$ Department of Breast \\ Cancer, The Affiliated Hospital of Qingdao University, Qingdao, China; ${ }^{6}$ Department of Breast Cancer, The First Hospital of China Medical \\ University, Shenyang, China \\ Contributions: (I) Conception and design: P Fu; (II) Administrative support: Z Jiang; (III) Provision of study materials or patients: S Wang; (IV) \\ Collection and assembly of data: J Li; (V) Data analysis and interpretation: S Wang; (VI) Manuscript writing: All authors; (VII) Final approval of \\ manuscript: All authors. \\ Correspondence to: Peifen Fu. Department of Breast Cancer, The First Affiliated Hospital, Zhejiang University, School of Medicine, Hangzhou, China. \\ Email: fupeifen@hotmail.com.
}

Background: The efficacy of endocrine therapy in breast cancer patients expressing low levels (1-9\%) of estrogen receptor (ER) is controversial, and there is limited clinical information about these patients.

Methods: Patients diagnosed with stage I to III primary invasive breast cancer with low ER expression between January 2008 and December 2016 from six breast centers in China were recruited for this study.

Results: Among the total of 17,216 patients, 457 (2.7\%) showed low ER expression. Of the 457 patients, $56.9 \%, 61.6 \%$, and $71.6 \%$ of tumors were human epidermal growth factor 2 (HER2) positive, progesterone receptor (PR) negative, and Ki-67 status $>20 \%$, respectively. Of these 457 patients, 112 patients received neoadjuvant chemotherapy, while 276 received adjuvant chemotherapy, and 170 patients received endocrine therapy. The total pathological complete response (pCR) rate was $26.8 \%$. The rate of patients who received endocrine therapy was center and time-dependent. ER expression, PR expression, Ki-67 status, and lymphovascular invasion (LVI) were associated with endocrine therapy decision-making using univariate logistic regression analysis. However, the adjustment for other covariates revealed that only the ER was significantly associated with the rate of patients receiving endocrine therapy. Compared to patients $<5 \%$ ERpositive tumors, patients with 5-9\% ER-positive tumors were significantly more likely to receive endocrine therapy (OR, 2.882; 95\% CI: 1.928-4.308; $\mathrm{P}<0.001)$. The median follow-up time was 30 months, and the five-year recurrence-free survival (RFS) rate was $85 \%$. Younger age and positive lymph nodes were associated with worse RFS. The survival rate was not significantly different between patients who received and those patients who did not receive endocrine therapy.

Conclusions: The 2010 ACSO/CAP recommendations increased in the proportion of patients with low ER expression receiving endocrine therapy. However, these patients do not appear to receive help from endocrine therapy.

Keywords: Breast cancer; estrogen receptor (ER); endocrine therapy; ER expression

Received: 25 May 2020; Accepted: 07 July 2020; Published: 30 July 2020.

doi: $10.21037 /$ tbcr-20-24

View this article at: http://dx.doi.org/10.21037/tbcr-20-24 


\section{Introduction}

Breast cancer is the most common malignancy and the leading cause of cancer deaths in women globally, with 1.7 million new cases and 535,000 associated deaths in 2016 (1); $60-75 \%$ of women with breast cancer have estrogen receptor (ER)-positive tumors. ER expression has been directly associated with the prognosis of breast cancer patients and is an essential predictor of endocrine therapy response (2-4).

In 2010, the American Society of Clinical Oncology (ASCO) and the College of American Pathologists (CAP) recommended that $\mathrm{ER}$ is considered positive if $\geq 1 \%$ of the tumor cells showed positive nuclear ER staining by immunohistochemistry (IHC). ASCO/CAP also recommended that endocrine therapy be considered in these patients with $\geq 1 \%$ ER-positive cells (5). However, some experts have questioned these recommendations because most of the prospective data detailing the correlation between the ER expression and treatment response was derived from ligand-binding assays (LBA) instead of traditional IHC (6-9). As most breast cancers patients present with ER-negative or strongly ER-positive $(\geq 10 \%)$ tumors, this recommendation has affected a small subset of patients who show low ER expression in the range of 1-9\% (10). Previously, Viale et al. retrospectively analyzed ER expression by IHC in 6,291 patients from the Breast International Group (BIG) 1-98 Trial and discovered that less than $1 \%$ of patients showed low ER expression (11). The small sample size of patients with low ER expression makes it difficult to obtain any dependable or statistically significant conclusions on optimal treatment strategies. For this reason, the ASCO/CAP recommends that oncologists discuss the benefits and risks of endocrine therapy with the patients to aid them in making an informed decision regarding treatment options (5).

As of now, there is limited information about the effect of endocrine therapy in breast cancer patients with low ER expression, and unfortunately, we have minimal clinical information about this subgroup of patients. In this study, we analyzed the clinicopathological features and treatment response to endocrine therapy in breast cancer patients with low ER expression. We looked to determine whether the ASCO/CAP guidelines impact the selection of patients receiving clinical endocrine therapy in China and to uncover which clinicopathological features are important considerations for physicians during treatment planning. Also, we evaluated the potential prognostic and predictive roles of low ER expression in breast cancer patients. In the future, these findings may allow physicians to make better treatment recommendations in breast cancer patients with low ER expression, which could lead to improved patient outcomes around the world. We present the following article in accordance with the STROBE reporting checklist (available at http://dx.doi.org/10.21037/tbcr-20-24).

\section{Methods}

\section{Ethical approval and informed consent}

This study was reviewed and approved by the Ethics Committees of the 307 Hospital of People's Liberation Army, the First Affiliated Hospital of Zhejiang University, the Affiliated Hospital of Qingdao University Medical College, the Fourth Hospital of Hebei Medical University, the First Affiliated Hospital of China Medical University, and the Jiangsu Province Hospital. The trial was conducted in accordance with the Declaration of Helsinki (revised 2013) and the Harmonized Tripartite Guideline for Good Clinical Practice from the International Conference on Harmonization.

\section{Patient selection}

A multi-institutional registry consisting of 17,216 patients with breast cancer who were diagnosed from January 2008 to December 2016 in China was set up. We identified patients who were diagnosed with stage I to III primary invasive breast cancer with low ER expression (1-9\%). Patients were excluded from this study when the IHC-based ER expression data were missing. A total of 457 patients were included in the final analysis. All pathologic specimens were reviewed by dedicated breast pathologists. The IHC and fluorescence in situ hybridization (FISH) analyses were performed using the same standard procedures described in the ASCO/CAP guidelines.

\section{Clinicopathological features}

Clinicopathological features were obtained from each patient, which included age, menopausal status, clinical stage, tumor size, nodal status, lymphovascular invasion (LVI), progesterone receptor (PR) expression, neoadjuvant chemotherapy, adjuvant chemotherapy, and adjuvant endocrine therapy. Pathological complete response (pCR) was defined as there being no invasive carcinoma in the breast 
or tumor in the axilla at the time of surgery. Recurrencefree survival (RFS) was calculated from the date of the initial diagnosis to the date of the first local or distant recurrence.

\section{Statistical analysis}

The clinicopathological features were tabulated and compared between the human epidermal growth factor 2 (HER2)-positive and HER2-negative groups using the Chi-square test and Fisher's exact test. Multivariate logistic regression models were used to investigate the association between clinicopathological variables and endocrine therapy for decision-making purposes. Kaplan-Meier curves were used to estimate the survival outcomes for all patients, and the log-rank test used to compare RFS between patients with and without endocrine therapy. The Cox proportional hazards model was used to determine the effects of prognostic factors on survival outcomes. SPSS Version 19.0 (IBM, Chicago, IL, USA) was used for the statistical analysis, and $\mathrm{P} \leq 0.05$ was considered statistically significant.

\section{Results}

\section{Patients and clinicopatbological characteristics}

Out of the 17,216 patients, only 457 (2.7\%) patients had low ER expression. The average percentage of ER expression in breast cancer patients with low ER expression was $3.8 \%$, with 267 patients having <5\% ER-positive cells and 190 patients having 5-9\% ER-positive cells. Also, 260 patients were HER2-positive, 185 patients were HER2negative, and twelve patients were HER2-unknown. In the HER2-positive group, 138 patients received Trastuzumabtargeted therapy, of which 29 received neoadjuvant Trastuzumab-targeted therapy. Also, 119 patients did not receive Trastuzumab-targeted therapy, and three patients were unknown.

The clinicopathological characteristics of the patients and tumors are summarized in Table 1. For all patients, the mean age at diagnosis was 49 years (range, 25-80 years). Most patients were premenopausal $(51.6 \%)$, and stage II was the most common clinical stage (55.6\%). Only $38.7 \%$

Table 1 Patient clinicopathological characteristics

\begin{tabular}{|c|c|c|c|c|}
\hline Variable & Total $(\mathrm{n}=457), \mathrm{n}(\%)$ & HER2-positive (n=260), n (\%) & HER2-negative ( $n=185), n(\%)$ & $P$ value \\
\hline Age, mean [range], years & 49 [25-80] & 49 [25-66] & 49 [28-61] & \\
\hline$>50$ & $229(50.1)$ & $131(50.4)$ & $91(49.2)$ & 0.8 \\
\hline$\leq 50$ & 228 (49.9) & $129(49.6)$ & $94(50.8)$ & \\
\hline \multicolumn{5}{|l|}{ Menopausal status } \\
\hline Premenopausal & $236(51.6)$ & $141(54.2)$ & $89(48.1)$ & 0.2 \\
\hline Postmenopausal & $221(48.4)$ & $119(45.8)$ & $96(51.9)$ & \\
\hline \multicolumn{5}{|l|}{ Clinical TNM stage } \\
\hline I & $110(24.1)$ & $52(20.0)$ & $52(28.1)$ & 0.09 \\
\hline II & $254(55.6)$ & $148(56.9)$ & $100(54.1)$ & \\
\hline III & $85(18.6)$ & $53(20.4)$ & $32(17.3)$ & \\
\hline Unknown & $8(1.8)$ & $7(2.7)$ & $1(0.5)$ & \\
\hline \multicolumn{5}{|l|}{ Clinical tumor size $(\mathrm{cm})$} \\
\hline $\mathrm{T} 1$ & $174(38.1)$ & $87(33.5)$ & $80(43.2)$ & 0.16 \\
\hline T2 & $244(53.4)$ & $146(56.2)$ & $93(50.3)$ & \\
\hline T3 & $27(5.9)$ & $17(6.5)$ & $10(5.4)$ & \\
\hline T4 & $5(1.1)$ & $4(1.5)$ & $1(0.5)$ & \\
\hline Unknow & $7(1.5)$ & $6(2.3)$ & $1(0.5)$ & \\
\hline
\end{tabular}

Table 1 (continued) 
Table 1 (continued)

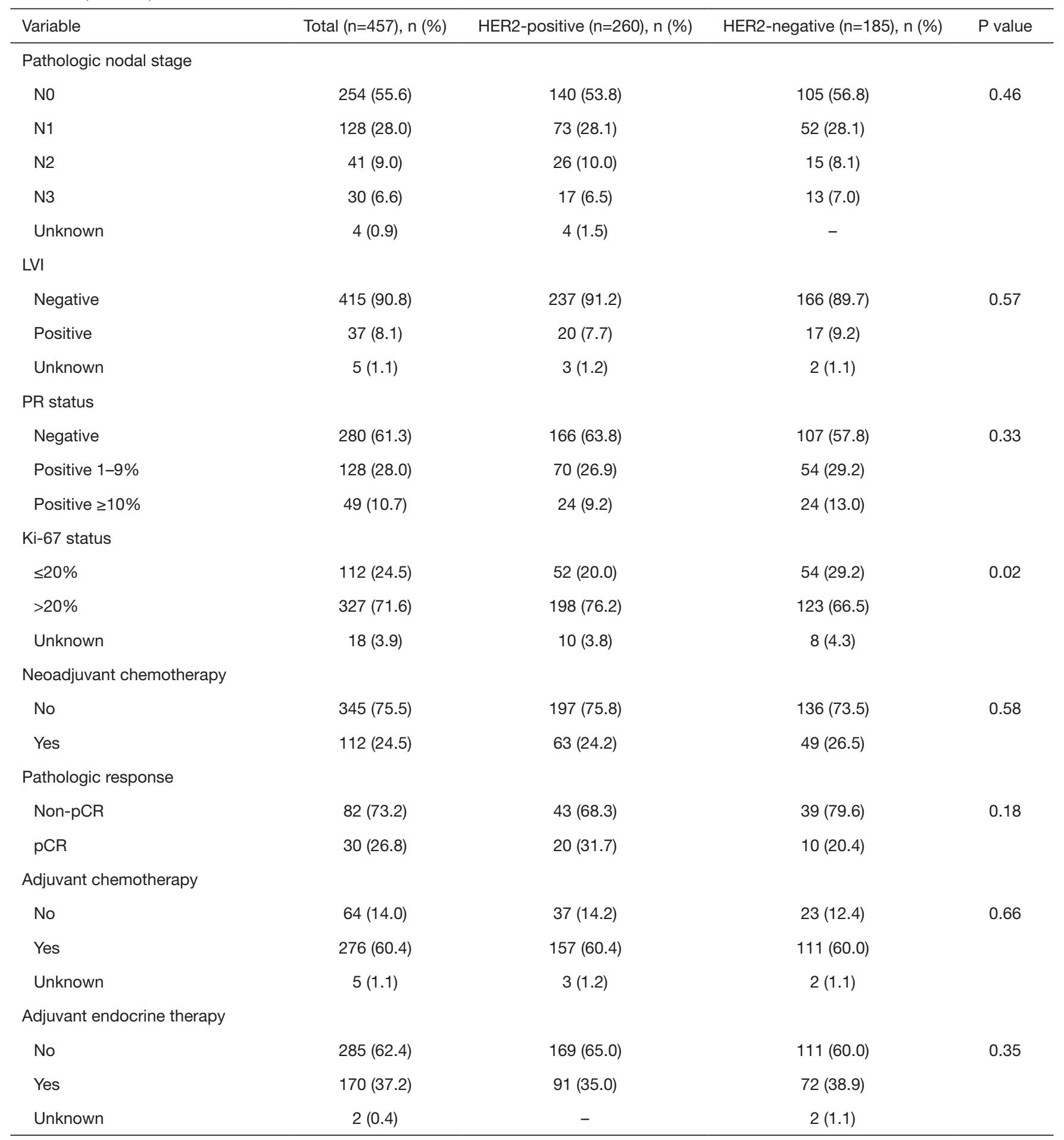

pCR, pathological complete response; HER2, human epidermal growth factor 2. 


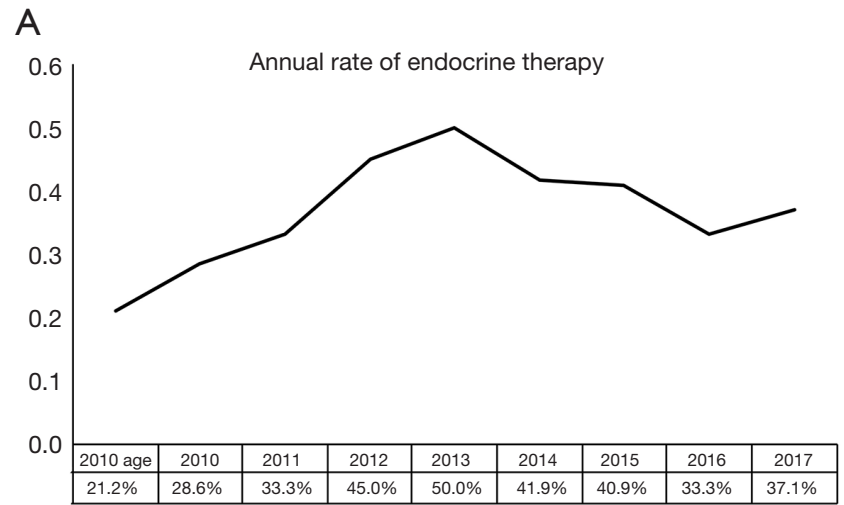

\section{B}

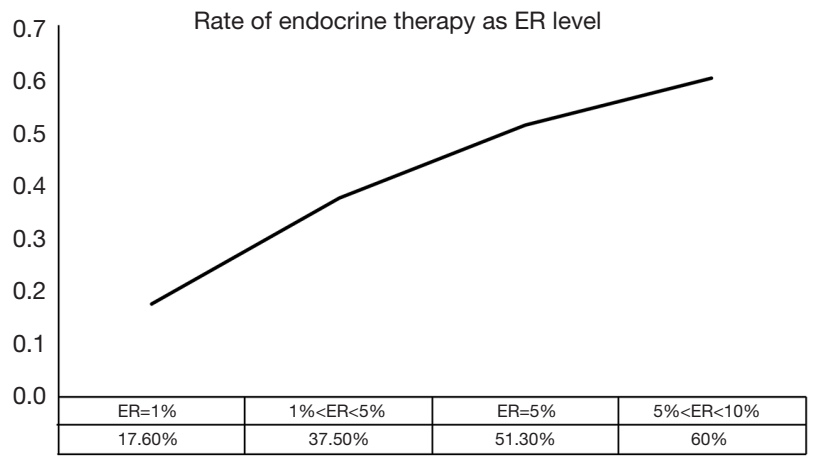

Figure 1 Rates of patients receiving endocrine therapy. (A) The annual rate of patients receiving endocrine therapy, and (B) the association between the rate of patients who received endocrine therapy and ER expression levels. ER, estrogen receptor.

of patients were PR-positive. The majority (71.6\%) of patients showed $>20 \% \mathrm{Ki}-67$ expression, while $33 \%$ of patients had $\geq 50 \% \mathrm{Ki}-67$ expression. When comparing the HER2-positive and HER2-negative groups, the HER2positive group showed higher (>20\%) Ki-67 levels (76.2\% vs. $66.5 \%, \mathrm{P}=0.02)$. However, the other characteristics, including age, stage, tumor size, nodal status, LVI, and PR status, were similar between the HER2-positive and HER2negative groups.

In total, 112 patients received neoadjuvant chemotherapy, and 276 patients received adjuvant chemotherapy, which in combination means that $85 \%$ of patients received chemotherapy. Of the 388 patients who received chemotherapy, $90 \%$ of patients received the combination of anthracycline (A) and taxol (T) regimens. There was no difference in the protocol of chemotherapy between the HER2-positive and HER2-negative groups. The total pCR rate was $26.8 \%$ for all patients. However, the pCR rate in the HER2-positive group (31.7\%) was slightly higher than the HER2-negative group (20.4\%), yet the difference was not statistically significant $(\mathrm{P}=0.18)$. Further analysis revealed that in the HER2-positive group, 29 patients received both neoadjuvant chemotherapy and neoadjuvant Trastuzumab-targeted therapy. Of the 29 patients, 14 (48.3\%) had $\mathrm{pCR}$, and the $\mathrm{pCR}$ rate was significantly higher than when compared with the HER2-negative group $(\mathrm{P}=0.01)$. Also, it was higher than those patients who did not receive neoadjuvant Trastuzumab-targeted therapy in the HER2positive group $(\mathrm{P}=0.009)$.

\section{Endocrine therapy in breast cancer patients with low ER expression}

In this study, $37.2 \%$ of patients with low ER expression received endocrine therapy, and there was no statistically significant difference between the HER2-positive and HER2-negative groups. The percentage of patients receiving endocrine therapy varied between the six centers, ranging from $4 \%$ to $55 \%(\mathrm{P}<0.05)$. The annual rate of patients receiving endocrine therapy is shown in Figure $1 A$. Before the updated ASCO/CAP guidelines released in 2010, only $25.7 \%$ of patients with low ER expression received endocrine therapy. The proportion of low ER-expressing breast cancer patients receiving endocrine therapy increased after 2010. In 2013, 50\% of these patients received endocrine therapy, which was significantly higher than the rate before $2010(\mathrm{P}<0.05)$. However, the proportion declined after 2013. ER expression, PR expression, Ki-67 status, and LVI were found to be associated with the rate of patients receiving endocrine therapy using univariate logistic regression analysis (Table 2). These results show that the clinicopathological features are important considerations for physicians to consider when accessing endocrine therapy during treatment planning. After adjusting for other covariates, only ER was determined to be significantly associated with the rate of endocrine therapy. Compared with patients who had tumors that were $<5 \%$ ER-positive, patients with tumors that were 5-9\% ERpositive were significantly more likely to receive endocrine therapy (OR, 2.882; 95\% CI: 1.928-4.308; $\mathrm{P}<0.001)$. The association between ER-expression and the rate of patients who received endocrine therapy may be found in Figure $1 B$.

\section{Survival outcomes}

The median follow-up time was 30 months (range, 
Table 2 Univariate logistic regression analysis of endocrine therapy

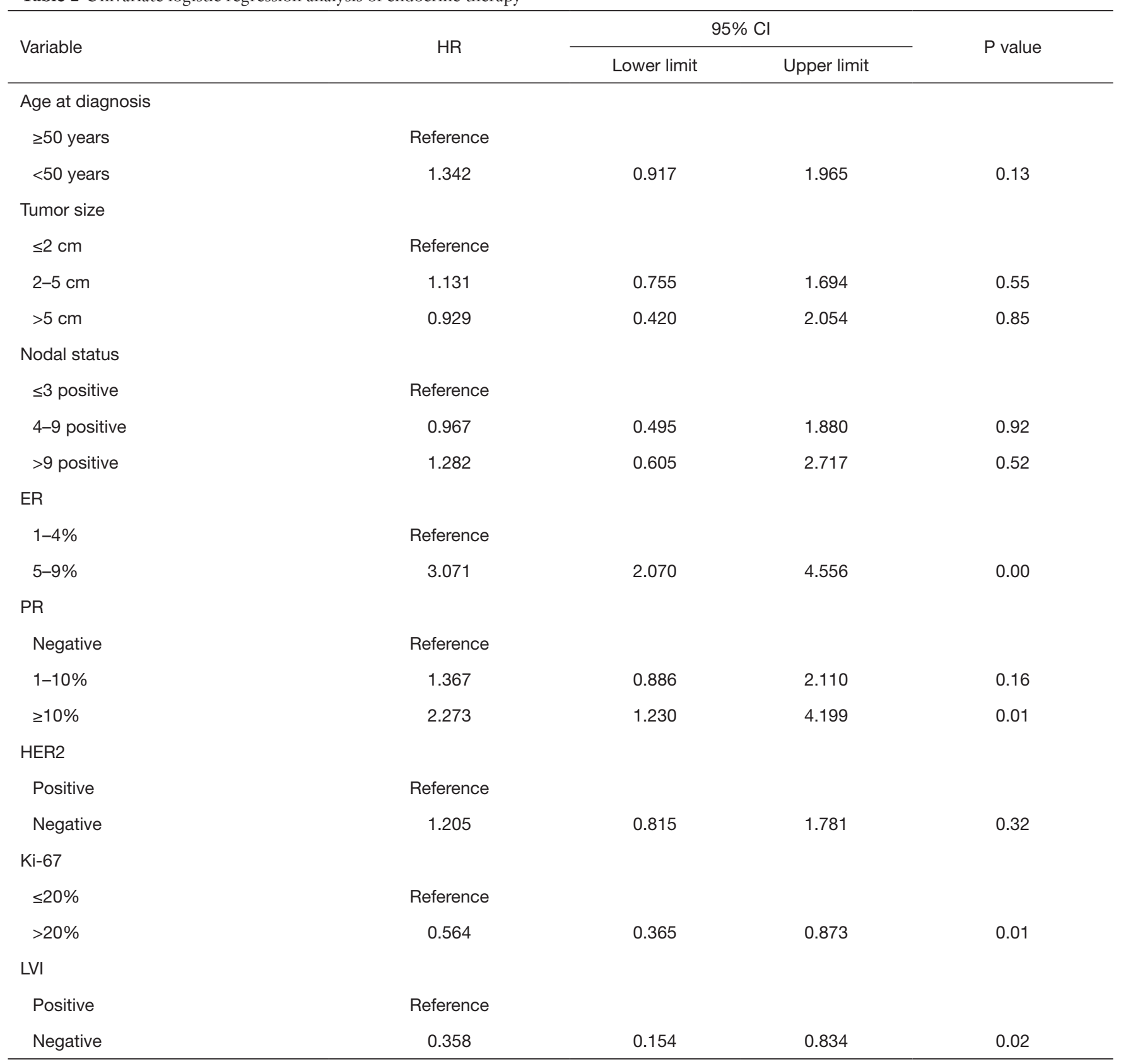

ER, estrogen receptor; PR, progesterone receptor; LVI, lymphovascular invasion; HER2, human epidermal growth factor 2.

3-122 months). The five-year RFS rate was recorded in $85 \%$ of the patients. As shown in Table 3, the univariate Cox proportional hazards model revealed that younger age ( $<50$ years), larger tumor size, and more positive lymph nodes were associated with increased risk of recurrence. Endocrine therapy did not significantly affect RFS
(Figure 2). The multivariate Cox proportional hazards model showed that only young age and positive lymph nodes were associated with worse RFS (age $<50$ vs. $\geq 50$ years, OR, 1.951; 95\% CI: $1.168-3.260 ; \mathrm{P}=0.01$; lymph node $\mathrm{pN} 2$ vs. pN0-1, OR, 2.405; 95\% CI: 1.264-4.574; $\mathrm{P}<0.001$; lymph node $\mathrm{pN} 3$ vs. pN0-1, OR, 4.994; 95\% CI: 2.754-9.056; $\mathrm{P}<0.001)$. 
Table 3 Univariate cox regression analysis of RFS

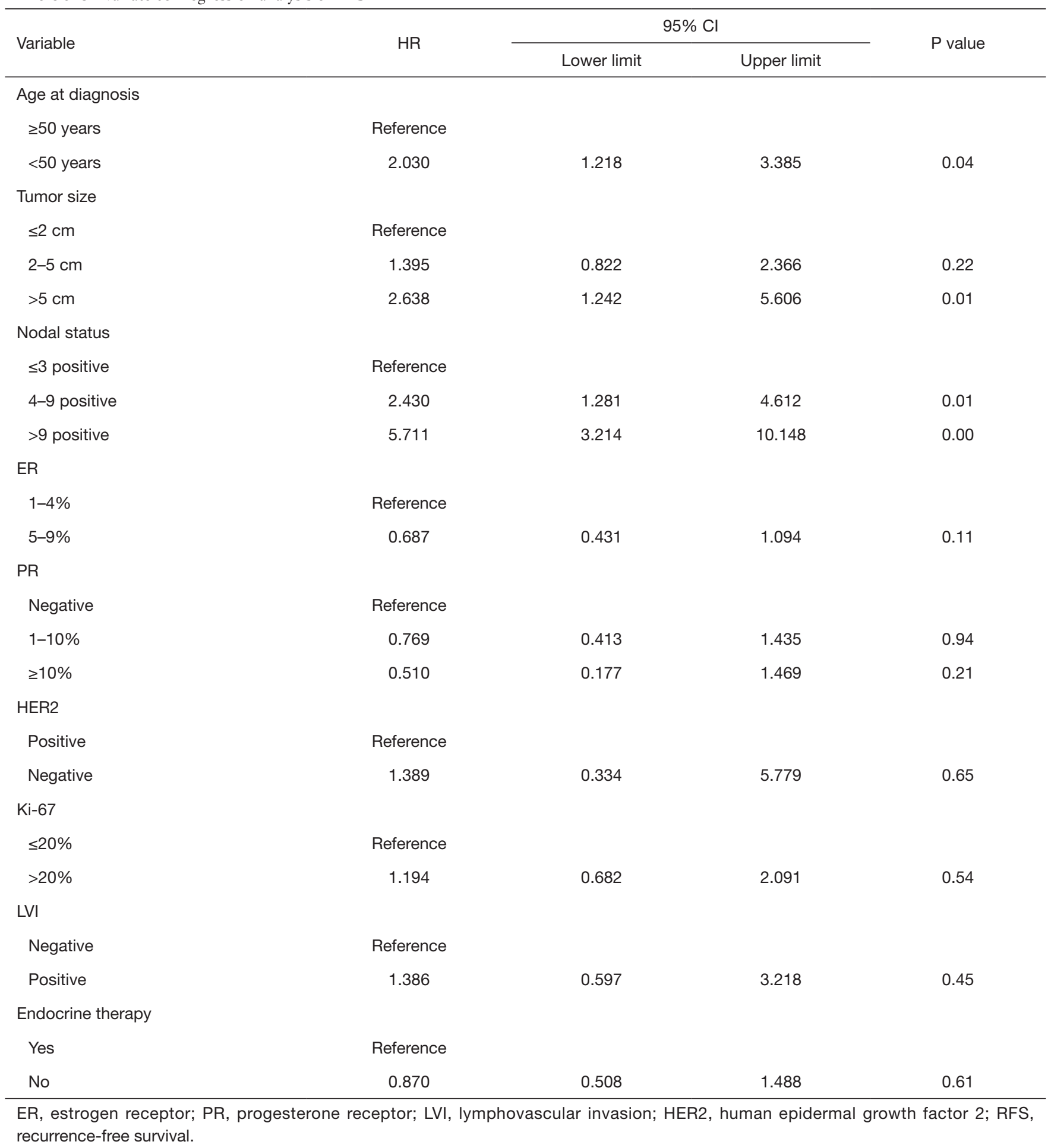



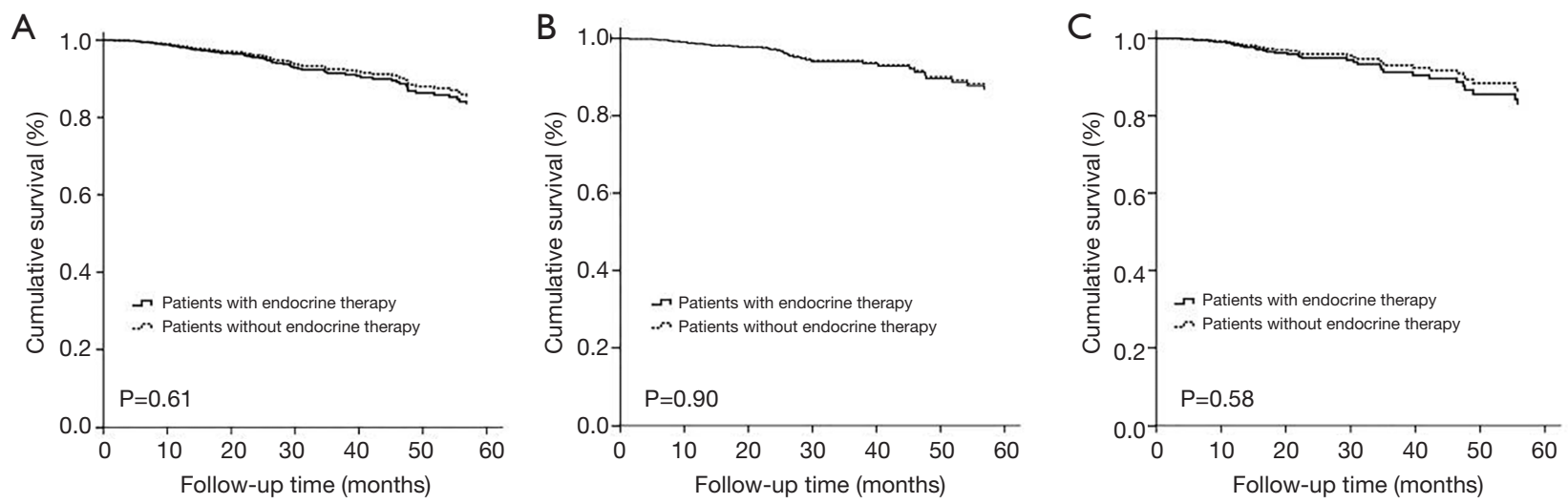

Figure 2 Comparison of recurrence-free survival (RFS) among patient who received and who did not receive endocrine therapy.

\section{Discussion}

The updated ASCO/CAP guidelines lowered the previous cutoff value of ER positivity, when assessed by IHC, and recommends that physicians consider endocrine therapy in patients whose breast tumors show $\geq 1 \%$ ER-positive cells (5). These results are made possible for breast cancer patients with low ER expression to receive endocrine therapy if desired. However, only a small subset of breast cancer patients fit this category. This study utilized the largest group of patients with low ER-expressing tumors up to now. In our study, 457 (2.7\%) patients showed low ER expression from multi-centers consisting of 17216 patients. This rate is similar to $2.6 \%(250 / 9,639)$, determined by $\mathrm{Yi}$ et al. (12), and 2.1\% (33/1,521) found by Regan et al. (9). However, higher rates have been reported. For example, Honma et al. found that $6.4 \%(73 / 1,149)$ of patients with highly invasive breast cancer low ER expression (13). Although the rates were different, what we can see from these studies is that this subgroup with ER low expression is rare, and the sample size may often be too small to draw reliable conclusions.

There have been several retrospective studies for patients with low ER expression, yet the prognosis and the endocrine responsiveness for these patients are often inconsistent. Some studies have reported that patients with low ER expression have a slightly better prognosis than ERnegative patients $(14,15)$ and that these patients can benefit from endocrine therapy (14). Also, several studies have shown that patients with low ER expression display similar characteristics and survival outcomes as patients with ER-negative tumors and that endocrine therapy did not impact the survival outcomes of these patients $(12,15-17)$.
In our study, we found that patients with low ER expression were young with a mean age of 49 years old. The majority of patients were stage II or III (74.2\%), with $56.9 \%$ of patients having tumors that were HER2-positive, $71.6 \%$ showing $>20 \% \mathrm{Ki}-67$ expression, in which $40 \%$ of Ki-67 $\geq 50 \%$. These clinicopathological features show that patients with low ER expression are strongly aggressive. Considering its clinicopathological features and prognosis, $85 \%$ of these patients received chemotherapy, and the vast majority $(90 \%)$ received $\mathrm{A}+\mathrm{T}$ for their chemotherapy regimens. The total pCR rate was $26.8 \%$ for patients with low ER expression patients in our study, with $20.4 \%$ in the HER2-negative group. It is similar to the pCR rate in TNBC (22-30\%) rather than hormone receptor-positive disease $(6.7-11 \%)(18,19)$. Also, Fujii et al. found that stage II or III HER2-negative primary breast cancer with ER expression of $<10 \%$ showed similar clinical outcomes to TNBC in terms of pCR (28.1\% vs. $26.3 \%)$, when compared with patients who showed $\geq 10 \%$ ER expression (7\%) (17). From the disputed results, prospective studies are needed to determine the best threshold for ER positivity.

In this study, we examined the impact of the updated ASCO/CAP guidelines on treatment planning in China for the first time. We found the proportion of patients receiving endocrine therapy increased after 2010, and that ER expression was the most crucial factor that physicians consider during the treatment planning process. Despite an increase in the number of patients receiving endocrine therapy, we found that only $37.2 \%$ of patients received endocrine therapy, and after 2013 , the rate of patients receiving endocrine therapy declined. We also found that the rate of endocrine therapy varied between the six 
centers. Currently, there is no uniform endocrine therapy plan because its clinical usefulness is still controversial for these patients. Many clinicians still use the cutoff value of $\geq 10 \%$ for considering patient eligibility, yet this study has shown that these patients are less likely to receive help from endocrine therapy.

While IHC is considered the standard for assessing ER expression, it may not be the most exact tool for determining treatment response. As an alternative, gene expression profiling may help guide clinical decisions in patients with low ER expression. Blueprint TM, an 80gene mRNA expression, and PAM 50 have been used for selecting patients with tumors more likely to respond to endocrine therapy, as they could identify luminal and basal tumors (20-22). These studies revealed that most tumors with low ER expression had molecular features like those of ER-negative tumors. However, other clinicopathological indicators such as PR should be considered, and chemotherapy may be more appropriate and vital for some patients in this subgroup.

There were two limitations to this study that should be considered. First, although this is the largest group of patients with low ER expression used to date, the group of patients is small. Secondly, this study was retrospective, and future studies using current patients may supply added insight into other factors that may potentially affect treatment response.

\section{Conclusions}

Our findings indicate that the 2010 ACSO/CAP recommendations increased the number of patients with low ER expression receiving endocrine therapy. However, endocrine therapy did not significantly improve the survival outcomes of these patients. More data is needed to determine the best ER expression cutoff value for treatment planning, considering the side effects of endocrine therapy. Prospective studies are needed for patients with low ER expression, and more exact methods are needed to evaluate ER levels and endocrine response.

\section{Acknowledgments}

Funding: The study was supported by the funding of the Chinese CSCO BC, the Natural Science Foundation of China (No. 81772845), and the Natural Science Doctoral Funding of Shandong province (No. ZR2019BH013 and ZR2017BH061).

\section{Footnote}

Reporting Checklist: The authors have completed the STROBE Checklist. Available at http://dx.doi. org/10.21037/tbcr-20-24

Data Sharing Statement: Available at http://dx.doi. org/10.21037/tbcr-20-24

Conflicts of Interest: All authors have completed the ICMJE uniform disclosure form (available at http://dx.doi. org/10.21037/tbcr-20-24). ZJ serves as an unpaid Editorin-Chief of Translational Breast Cancer Research. JL serves as an unpaid Managing Editor of Translational Breast Cancer Research. YY, YL, HW serve as the unpaid editorial board members of Translational Breast Cancer Research from Mar 2020 to Feb 2022. The other authors have no conflicts of interest to declare.

Ethical Statement: The authors are accountable for all aspects of the work in ensuring that questions related to the accuracy or integrity of any part of the work are appropriately investigated and resolved. The study was conducted in accordance with the Declaration of Helsinki (revised 2013) and the Harmonized Tripartite Guideline for Good Clinical Practice from the International Conference on Harmonization. This study was approved by the Institutional Review Board of The Affiliated Hospital of Qingdao University (QYFYKYLL 221311920). Informed consent was obtained from all individual participants included in the study.

Open Access Statement: This is an Open Access article distributed in accordance with the Creative Commons Attribution-NonCommercial-NoDerivs 4.0 International License (CC BY-NC-ND 4.0), which permits the noncommercial replication and distribution of the article with the strict proviso that no changes or edits are made and the original work is properly cited (including links to both the formal publication through the relevant DOI and the license). See: https://creativecommons.org/licenses/by-nc-nd/4.0/.

\section{References}

1. Global Burden of Disease Cancer Collaboration, Fitzmaurice C, Akinyemiju TF, et al. Global, Regional, and National Cancer Incidence, Mortality, Years of Life Lost, Years Lived With Disability, and Disability-Adjusted Life- 
Years for 29 Cancer Groups, 1990 to 2016: A Systematic Analysis for the Global Burden of Disease Study. JAMA Oncol 2018;4:1553-68.

2. Regan MM, Neven P, Giobbie-Hurder A, et al. Assessment of letrozole and tamoxifen alone and in sequence for postmenopausal women with steroid hormone receptorpositive breast cancer: the BIG 1-98 randomised clinical trial at 8.1 years median follow-up. Lancet Oncol 2011;12:1101-8.

3. Davies C, Pan H, Godwin J, et al. Long-term effects of continuing adjuvant tamoxifen to 10 years versus stopping at 5 years after diagnosis of oestrogen receptorpositive breast cancer: ATLAS, a randomised trial. Lancet 2013;381:805-16.

4. Burstein HJ, Temin S, Anderson H, et al. Adjuvant endocrine therapy for women with hormone receptorpositive breast cancer: american society of clinical oncology clinical practice guideline focused update. J Clin Oncol 2014;32:2255-69.

5. Hammond ME, Hayes DF, Dowsett M, et al. American Society of Clinical Oncology/College Of American Pathologists guideline recommendations for immunohistochemical testing of estrogen and progesterone receptors in breast cancer. J Clin Oncol 2010;28:2784-95.

6. Barnes DM, Harris WH, Smith P, et al. Immunohistochemical determination of oestrogen receptor: comparison of different methods of assessment of staining and correlation with clinical outcome of breast cancer patients. Br J Cancer 1996;74:1445-51.

7. Elledge RM, Green S, Pugh R, et al. Estrogen receptor (ER) and progesterone receptor (PgR), by ligand-binding assay compared with ER, $\mathrm{PgR}$ and $\mathrm{pS} 2$, by immuno-histochemistry in predicting response to tamoxifen in metastatic breast cancer: a Southwest Oncology Group Study. Int J Cancer 2000;89:111-7.

8. Thomson CS, Twelves CJ, Mallon EA, et al. Adjuvant ovarian ablation vs CMF chemotherapy in premenopausal breast cancer patients: trial update and impact of immunohistochemical assessment of ER status. Breast 2002;11:419-29.

9. Regan MM, Viale G, Mastropasqua MG, et al. Reevaluating adjuvant breast cancer trials: assessing hormone receptor status by immunohistochemical versus extraction assays. J Natl Cancer Inst 2006;98:1571-81.

10. Collins LC, Botero ML, Schnitt SJ. Bimodal frequency distribution of estrogen receptor immunohistochemical staining results in breast cancer: an analysis of 825 cases. Am J Clin Pathol 2005;123:16-20.

11. Viale G, Regan MM, Maiorano E, et al. Prognostic and predictive value of centrally reviewed expression of estrogen and progesterone receptors in a randomized trial comparing letrozole and tamoxifen adjuvant therapy for postmenopausal early breast cancer: BIG 1-98. J Clin Oncol 2007;25:3846-52.

12. Yi M, Huo L, Koenig KB, et al. Which threshold for ER positivity? a retrospective study based on 9639 patients. Ann Oncol 2014;25:1004-11.

13. Honma N, Horii R, Iwase T, et al. Proportion of estrogen or progesterone receptor expressing cells in breast cancers and response to endocrine therapy. Breast 2014;23:754-62.

14. Viale G, Regan MM, Maiorano E, et al. Chemoendocrine compared with endocrine adjuvant therapies for nodenegative breast cancer: predictive value of centrally reviewed expression of estrogen and progesterone receptors--International Breast Cancer Study Group. J Clin Oncol 2008;26:1404-10.

15. Balduzzi A, Bagnardi V, Rotmensz N, et al. Survival outcomes in breast cancer patients with low estrogen/ progesterone receptor expression. Clin Breast Cancer 2014;14:258-64.

16. Raghav KP, Hernandez-Aya LF, Lei X, et al. Impact of low estrogen/progesterone receptor expression on survival outcomes in breast cancers previously classified as triple negative breast cancers. Cancer 2012;118:1498-506.

17. Fujii T, Kogawa T, Dong W, et al. Revisiting the definition of estrogen receptor positivity in HER2-negative primary breast cancer. Ann Oncol 2017;28:2420-8.

18. Liedtke C, Mazouni C, Hess KR, et al. Response to neoadjuvant therapy and long-term survival in patients with triple-negative breast cancer. J Clin Oncol 2008;26:1275-81.

19. von Minckwitz G, Untch M, Blohmer JU, et al. Definition and impact of pathologic complete response on prognosis after neoadjuvant chemotherapy in various intrinsic breast cancer subtypes. J Clin Oncol 2012;30:1796-804.

20. Chia SK, Bramwell VH, Tu D, et al. A 50-gene intrinsic subtype classifier for prognosis and prediction of benefit from adjuvant tamoxifen. Clin Cancer Res 2012;18:4465-72. 
21. Iwamoto T, Booser D, Valero V, et al. Estrogen receptor (ER) mRNA and ER-related gene expression in breast cancers that are $1 \%$ to $10 \%$ ER-positive by immunohistochemistry. J Clin Oncol 2012;30:729-34.

doi: $10.21037 /$ tbcr-20-24

Cite this article as: Wang S, Li J, Jiang Z, Yin Y, Liu Y, Wang H, Teng Ye, Ma L, Song Y, Fu P. Breast cancer patients with low estrogen receptor expression gain no significant survival benefit from endocrine therapy: a real-world study from China. Transl Breast Cancer Res 2020;1:14.
22. Krijgsman O, Roepman P, Zwart W, et al. A diagnostic gene profile for molecular subtyping of breast cancer associated with treatment response. Breast Cancer Res Treat 2012;133:37-47. 